http://doi.org/10.15359/ree.12-2.2

\title{
CONSTRUCCIÓN DEL PERFIL PROFESIONAL DOCENTE DE SÉTIMO AÑO: RESPUESTA A UNA NECESIDAD ACTUAL
}

\author{
Magdalena Alfaro Rodríguez ${ }^{1}$ \\ Académica de la División de Educología del CIDE-Universidad Nacional \\ Heredia-Costa Rica \\ Alejandra Gamboa Jiménez ${ }^{2}$ \\ Académica de la División de Educación para el Trabajo del CIDE-Universidad Nacional \\ Heredia-Costa Rica \\ Susana Jiménez Sánchez ${ }^{3}$ \\ Académica de la División de Educología del CIDE-Universidad Nacional \\ Heredia-Costa Rica \\ Jorge Martín Pérez ${ }^{4}$ \\ Académico del INEINA del CIDE-Universidad Nacional \\ Heredia-Costa Rica \\ Andrea Ramírez González ${ }^{5}$ \\ Académica de la División de Educación Rural del CIDE-Universidad Nacional \\ Heredia-Costa Rica \\ Marie-Claire Vargas Dengo ${ }^{6}$ \\ Académica de la División de Educación Básica del CIDE-Universidad Nacional \\ Heredia-Costa Rica
}

Magíster en Educación con énfasis Docencia Universitaria de la Universidad Nacional. Licenciada en Música. Actualmente es académica de la División de Educología del CIDE, donde integra el tercer equipo del Proyecto Perfiles, Dinámicas y Desafíos de la Educación Costarricense.magdar613@hotmail.com

Licenciada en Enseñanza de la Matemática de la Universidad de Costa Rica y con estudios en Educación Primaria. Actualmente es académica de la División de Educación Rural del CIDE, donde integra el tercer equipo del Proyecto Perfiles, Dinámicas y Desafíos de la Educación costarricense. aramgo@costarricense.cr

Máster en Currículum e Instrucción de The George Washington University, USA. Actualmente es académica de la División de Educación Básica del CIDE, donde integra el tercer equipo del Proyecto Perfiles, Dinámicas y Desafíos de la Educación Costarricense. mariedclaire@yahoo.com 
Recibido: 9 de octubre, 2007 - Aprobado: 29 de noviembre, 2007

Resumen: El sétimo año es el nivel que presenta el mayor índice de deserción y de reprobación escolar, dentro de la educación secundaria de nuestro país. Ésta fue la principal razón por la que este grupo de investigadores se propuso construir el perfil profesional de los y las docentes de este grado escolar. Con base en una definición sobre las características de las personas que se dedican a la docencia, el concepto de lo que debe ser un perfil, las características etarias de los estudiantes de sétimo año y todos aquellos aspectos que ayudan a que los procesos de enseñanza y de aprendizaje resulten exitosos, se plantea en este escrito el perfil óptimo necesario de los y las docentes que tienen a cargo los grupos de sétimo año.

Palabras clave: Educación secundaria, sétimo año, perfil docente, deserción, permanencia, reprobación.

\begin{abstract}
In our country, the seventh grade is the level with the highest desertion and failure rate in high school. This was the main reason that motivated this group of researchers to define the teachers' professional profile at this level. Based on a definition of the teachers' characteristics, the concept of how a profile should be, the age features of seventh graders and all those aspects that benefit the teaching-learning processes, the optimum and required profile of the seventh graders' teachers is proposed.
\end{abstract}

Key words: High School, seventh grade, teachers' profile, desertion, permanence, school failure.

\title{
Sinopsis de la situación actual de la educación secundaria costarricense
}

El estado de la educación secundaria es motivo de preocupación debido a las alarmantes estadísticas de los últimos tres años referidas a la deserción, repitencia y calidad en el III Ciclo y en la educación diversificada. Según el Duodécimo Informe del Estado de la Nación (2006), la expulsión se incrementó en el III Ciclo, al pasar de 10,4\% en el 2003, a 11,6\% en el 2004 y 12,5\% en el 2005. En sétimo año, estas cifras alcanzaron $16,6 \%, 18,3 \%$ y $19,2 \%$, respectivamente. Las tasas más elevadas siguen registrándose en las etapas de transición, es decir, al ingreso a secundaria en sétimo año y al inicio de la modalidad diversificada en décimo año.

Lo anterior incide en el destino de cientos de adolescentes que, no cuentan durante esta etapa tan importante, con un proyecto de vida congruente con los derechos que, como ciudadanos menores de edad tienen. Además, se suma a esta situación, el gasto millonario que le corresponde al país invertir por esta amenazante realidad. Se habla de una pérdida económica de 51000 millones de colones aproximados por año (representados en el 0,5\% del 
Producto Interno Bruto), por causa de la deserción y la reprobación, según los datos estimados por el Duodécimo Informe del Estado de la Nación (2006). Como señala Trejos en este Informe, no todo lo que se invierte en cada estudiante que pierde el curso lectivo y lo repite se desaprovecha, puesto que se construye algún aprendizaje y se desarrollan ciertas destrezas en la población estudiantil; sin embargo, lo real es que el proceso no se completó, y, en esa medida, la inversión del Estado obtiene un menor rendimiento.

Esta problemática no se podrá solucionar con sólo describir un diagnóstico de la educación en Costa Rica, sino que se hace necesario profundizar en las diferentes aristas que intervienen en este fenómeno. Por tal razón, es motivo de investigación para detectar las causas de esta problemática. Ruiz (2006), hace referencia a que existen algunas variables que intervienen en que la población de secundaria deserte, entre ellas "la incorporación de los jóvenes en la vida productiva, ya sea en el trabajo asalariado o en trabajos domésticos familiares, la escolaridad de la familia y el contexto de apoyo familiar al estudiante, problemas particulares que afectan la vida personal de los estudiantes, como por ejemplo: embarazos, drogadicción, estrés familiar, cambios drásticos en las condiciones de vida, etc." (p. 26).

Conocer solamente el escenario negativo de la educación, no ofrece soluciones para mejorar su calidad; por lo que se considera que uno de los principales aspectos de interés, es entender el fenómeno educativo como un todo. Los acuerdos y los desacuerdos en las alternativas de solución existentes para una calidad de la educación están a plena luz, basta con visitar una institución educativa para darse cuenta de las iniciativas profesionales gestadas en cada una de ellas; claro está, existen grandes desafíos que, con el tiempo, aparecen y otros que se han perpetuado con el pasar del tiempo; no obstante, éste es tema de otro ensayo.

Por estas razones, muchos docentes en el ámbito mundial, preocupados por la problemática educativa, han encontrado alternativas para solucionar estas situaciones, de modo que los estudiantes tengan una mejor formación. Una de ellas podría ser la instauración del bachillerato internacional, con el que se podría obtener un perfil de salida del estudiante de secundaria, adaptable a cualquier nivel escolar de las instituciones educativas de los países que lo tienen inscrito. Por supuesto, en el entendido de que esto también es una iniciativa entre 
otras, que brindaría al estudiantado ciertos saberes necesarios para desenvolverse en la sociedad en la que está inmerso.

\section{Características del sétimo año}

El tercer ciclo de la Educación General Básica inicia en el nivel de sétimo año en las instituciones de enseñanza media. En este nivel educativo, para instituciones académicas diurnas, el currículo propone el estudio de asignaturas fundamentales y especiales e idiomas. En total, son 14 asignaturas que deben impartirse con un número determinado de lecciones semanales para cada una, a saber:

\begin{tabular}{|l|c|}
\hline \multicolumn{1}{|c|}{ Asignatura } & $\mathbf{N}^{\circ}$ de lecciones \\
\hline Ciencias & 5 \\
\hline Matemática & 6 \\
\hline Español & 5 \\
\hline Estudios sociales & 4 \\
\hline Cívica & 2 \\
\hline Inglés & 3 \\
\hline Francés & 3 \\
\hline Artes plásticas & 2 \\
\hline Educación musical & 2 \\
\hline Artes industriales & 3 \\
\hline Educación para el hogar & 3 \\
\hline Educación física & 2 \\
\hline Orientación & 1 \\
\hline Lección guía & 1 \\
\hline
\end{tabular}

Fuente: Costa Rica. Ministerio de Educación Pública (2001).

Para la implementación y el desarrollo de cada una de estas asignaturas, se contratan profesores graduados (en la mayoría de los casos) de las carreras Enseñanza de..., provenientes de las diferentes universidades públicas y privadas de nuestro país; por lo que, se supone, se 
cuenta con un docente especialista en un área disciplinar que no sólo tiene un amplio conocimiento de su disciplina, sino que, también, posee la formación pedagógica necesaria para lograr procesos de enseñanza y de aprendizaje satisfactorios, vinculados al programa de estudio correspondiente.

El o la docente, en su lección, desarrolla contenidos propios de su materia, en ella se conjugan habilidades cognitivas, métodos, técnicas, procedimientos, actitudes y valores enfocados, finalmente, a la formación integral del estudiante; de ahí que el proceso de organización, de planeamiento y de ejecución de la lección son específicos, pues se fundamentan en una naturaleza epistemológica de un área disciplinar que se diferencia de las otras por sus particularidades. Por tanto, las dinámicas de una lección de Matemática y de Español, por ejemplo, no son las mismas, porque se busca alcanzar diferentes saberes conceptuales, procedimentales y actitudinales.

Por lo anterior, el estudiantado de sétimo año se somete a un currículo compuesto por todas las asignaturas correspondientes a ese nivel, con docentes especialistas para cada materia y, a su vez, con el requerimiento de cumplir con actividades y tareas específicas que le exigen realizar.

\section{Entre el sexto y el sétimo años}

El ingreso al sétimo año de estudios formales se produce, generalmente, en un momento de tránsito en el desarrollo del niño hacia la adolescencia, al que se atribuyen inseguridades, indefiniciones, carencias, identidad ambigua y necesidad de asentamiento interior y social, con múltiples manifestaciones que consumen gran parte de las capacidades del sujeto.

Según Andersen (2007), citado por Castro, Díaz, Fonseca, Moraga, Umaña y Ruíz (2007), los adolescentes evidencian:

1. Mayor capacidad para comprender conceptos abstractos, filosofías y los conceptos de derechos y privilegios.

2. Capacidad para cuestionar viejos valores, sin sentir que pierden su identidad. 
3. Desarrollar un sentido de identidad y propósito más maduro.

4. Establecer y mantener relaciones interpersonales más satisfactorias, aprendiendo a compartir sin inhibiciones o miedos.

Pudiera ser contradictorio que en esta etapa, trascendental y cambiante de su desarrollo, los seres humanos se vean sometidos a cambios bruscos en la casi única institución social, fuera de su familia, que les puede haber promovido un sentido de pertenencia: la escuela.

Durante un largo período de más de seis años, los niños y las niñas conviven en un segundo hogar, en este caso más diverso, pero con estabilidad normativa, grupal y afectiva, tanto con sus coetáneos, como con un relativamente reducido grupo de maestras y maestros, con los que, en general, se llegan a desarrollar relaciones bastante familiares.

La conclusión de los estudios de nivel primario tiene un reconocimiento social y familiar destacado que reciben niñas y niños, resultante de la primera interacción institucional sistemática y, significativamente, prolongada que han tenido.

Como premio a este instante de éxito, producto de todo un proceso de estabilización física y emocional, la sociedad les impone nuevos cambios bruscos, incertidumbres y expectativas, con su arribo al nivel secundario de enseñanza. El sétimo se exhibe como un verdadero obstáculo difícil de vencer, que compite y puede opacar con un rotundo fracaso, el reciente éxito de la graduación del sexto, que representó más que un simple grado, todo un ciclo escolar.

Es así como el estudiante de sétimo año se expone a una serie de transformaciones que repercuten en su vida personal y que, por ende, caracterizan este grado de escolaridad; es el tiempo de transición de la primaria a la secundaria provista de una gama de aspectos y situaciones que el y la adolescente tienen que atender, comprender y ajustarse al momento. Al respecto, Castro et al (2007), en su investigación sobre Factores de éxito en la transición de sexto a sétimo, concluyen que el proceso de transición evidencia: 
1. Un cambio brusco de primaria a secundaria.

2. Las principales dificultades identificadas por los y las estudiantes fueron: los cambios de horario, la cantidad de materias y de profesoras(es); el bajo nivel de comprensión de las materias básicas y el temor a incursionar en nuevos grupos sociales. El factor que más conduce al fracaso, según ellos y ellas, es la falta de interés por el estudio; la indisciplina y el bajo rendimiento. No hacen ninguna referencia a factores económicos, mientras que para los y las docentes lo económico sí es relevante, tanto como la motivación o el desinterés y los problemas familiares.

3. Para los padres y las madres, los factores más relevantes son el apoyo familiar, el interés de ellos y ellas por el estudio y la motivación que pueden ofrecer los y las docentes. En síntesis, estudio, apoyo y dedicación constituyen los elementos claramente identificados como críticos para propiciar el éxito y la permanencia en el sistema educativo.

4. A los estudiantes de sexto grado lo que más les preocupa del colegio es fracasar en los estudios, el nivel de exigencia y la adaptación a las normas sociales, y los posibles problemas de violencia. La mayoría de los alumnos y las alumnas tiene una visión positiva, aunque les gustaría que fuera más alegre y divertido, y más fácil. Se reflejan aquí actitudes y valores complejos, ya que será difícil mejorar la calidad de la educación aunque se reconozca la importancia del estudio y la dedicación; pareciera que, en el fondo, lo que se quisiera establecer es lo que dicen los estudiantes de sexto grado.

5. En relación con las actividades realizadas para preparar a los estudiantes para el ingreso a la secundaria se concentraron en prácticas para los exámenes, actividades de refuerzo y recuperación, y folletos o copias; es decir, la gran mayoría fueron academicistas.

En su ambivalencia, la aprobación del sétimo año no es más que sobrepasar un sofocón, pero de un grado más, sin reconocimiento social alguno, frente al fracaso que representa reprobarlo, lo que, unido a lo anterior, puede inducir a la deserción. Es aquí donde la labor del profesorado, es trascendental para evitar la exclusión del estudiantado del sistema educativo. 


\section{El y la docentes en los procesos de enseñanza y de aprendizaje}

En los procesos de enseñanza y de aprendizaje, el profesorado, necesariamente, deberá establecer espacios de reflexión, de ejercicios metacognitivos por parte de los estudiantes y de metadidáctica. Son los y las docentes los que por medio de la introspección pueden mejorar su quehacer profesional. Con respecto a esto, Shön (1998), señala que "la reflexión de un profesional puede servir como correctivo del sobreaprendizaje. A través de la reflexión puede hacer emerger y criticar las comprensiones tácitas que han madurado en torno a las experiencias repetitivas de una práctica especializada, y puede dar un nuevo sentido a las situaciones de incertidumbre o únicas que él pueda permitirse experimentar" (p. 66).

En este proceso, el profesorado debe implementar los cambios pertinentes. Tal vez, en este momento histórico, no es tan importante qué aprende el o la estudiante, sino cómo aprende y desde esta perspectiva, es necesario que el y la docentes tomen en cuenta las características, el nivel de madurez emocional y los intereses de los y las estudiantes.

En el caso particular de la secundaria (de la que se ocupa este ensayo), podría ser que los y las docentes, tengan muy buenas y novedosas estrategias de enseñanza y de aprendizaje, muy buenos métodos y estrategias didácticas; pero si no se toman en cuenta algunos factores específicos de los adolescentes, como el idealismo y el carácter crítico, la tendencia a discutir, la indecisión, la hipocresía aparente, la autoconciencia y la suposición de ser especial e invulnerable (Elkind, citado por Papalia, Wendkos y Duskin, 2005), podría ser que no se tenga éxito en la labor docente.

Por otro lado, el clima de aprendizaje debe estar cargado de relaciones interpersonales afectuosas y cálidas, no sólo entre el estudiantado, sino entre éste y el profesorado, de modo que faciliten el aprendizaje. Con respecto a esto, Díaz y Hernández (2002), anotan algunas áreas de competencia, de conocimientos, así como características de un buen docente, dentro de los que se encuentran: 
1. Conocimiento teórico suficiente profundo y pertinente acerca del aprendizaje, el desarrollo y el comportamiento humano.

2. Despliegue de valores y actitudes que fomenten el aprendizaje y las relaciones humanas genuinas.

3. Dominio de los contenidos o materias que enseña.

4. Control de estrategias de enseñanza que faciliten el aprendizaje del alumno y lo hagan motivante.

5. Conocimiento personal práctico sobre la enseñanza (p. 4).

Asimismo, es el profesorado el que promueve aprendizajes significativos en los y las adolescentes, con los que ellos y ellas sientan motivación, y que no sólo les permita tener un mayor nivel de razonamiento, sino ser mejores personas, que los prepare para la vida y que los comprometa con un desarrollo humano integral propio. De acuerdo con Díaz y Hernández (2002), el aprendizaje significativo es más importante y deseable que el repetitivo, en lo que se refiere a situaciones académicas, ya que posibilita la adquisición de muchos conocimientos integrados, coherentes, estables y con sentido para los alumnos. Las prácticas de enseñanza constituyen, entonces, la mediación final que marca las posibilidades de lo que, realmente, pueden aprender los y las discentes, y el espacio en que se define la calidad de la educación.

Estas características y muchas otras son fundamentales en la construcción de un perfil profesional idóneo para los y las docentes de sétimo año, si se quiere el mejoramiento de los procesos educativos en secundaria.

\section{¿Qué es el perfil del docente?}

El perfil docente es una de las variables fundamentales que intervienen en la interrelación estudiantes-docentes e incide en la dinámica del proceso educativo y, por tanto, en su calidad.

El concepto perfil de desempeño se relaciona con una serie de características personales y conductuales que, en la época actual, se rescatan a partir de la valoración de la diversidad, la 
diferenciación de particularidades humanas, las capacidades, los valores, las actitudes, los estilos cognitivos y las pautas de comportamiento.

Se entiende por perfil docente, los rasgos específicos que caracterizan al educador. Dentro de una concepción integral, el perfil conjuga peculiaridades relacionales, cognitivas y emocionales que se expresan en el ámbito educativo. Los rasgos positivos conforman un perfil óptimo que impacta, de manera consecuente, al estudiantado; razón por la cual, pueden convertirse en un orientador para la selección y la contratación laboral, la calificación, la evaluación y la promoción de los(as) docentes.

Un óptimo perfil docente concibe tales rasgos y características, como cualidades que posibilitan al educador y a la educadora afrontar, con acierto, las situaciones del entorno.

Si bien muchos de estos atributos pueden ser innatos, como la vocación, otros se llegan a aprender y a desarrollar. Sin embargo, el énfasis en algunos rasgos, como por ejemplo en el intelecto, no asegura un óptimo desempeño docente. De aquí la importancia y las implicaciones del tema para la formación, la capacitación continua y el ejercicio del rol profesional docente.

Los perfiles profesionales docentes han sido un tema de estudio por más de tres décadas (Guedez, 1980; Grell, 1976 y otros, citados por Valenzuela, 1996). Según Valenzuela, Guedez describe el perfil profesional como "el conjunto de características pretendidas por el empleador, las cuales deben reflejar las exigencias del mercado ocupacional, en términos de los requisitos que definan las habilidades, las destrezas, los rasgos de personalidad, la conformación física y el nivel de educación inherente al desempeño profesional" (p. 7). En esta línea, dice Valenzuela que se hace necesaria una propuesta de perfil docente para la enseñanza media que considere valores curriculares como el vocacional, el cultural, el científico, el psicopedagógico y el experiencial.

La sociedad actual demanda un perfil de educador actualizado con la época, con los contextos social, institucional, regional y global, conocedor de lo que pasa en el mundo. Con capacidad crítica, con creatividad, que se eduque permanentemente y que trabaje en equipo, que comprenda el contexto y el fenómeno educativo, con disposición de integrarse como miembro responsable a la gestión educativa. Además, debe responder a los planteamientos de las políticas nacionales en materia educativa, las cuales, en nuestro país, sugieren un perfil de 
persona-ciudadano que se espera, en relación con el momento histórico de la sociedad costarricense.

Tradicionalmente, se ha considerado capacitado para enseñar a quien manifiesta algunas competencias profesionales específicas. Sin embargo, actualmente, definir los distintos criterios para conformar un adecuado perfil docente no es una tarea sencilla, por cuanto se debe contemplar una serie de aspectos, requisitos y cualidades que respondan tanto a las necesidades de la sociedad y la época actual, como a las de la población particular para la que se define ese perfil.

En esta época de crisis de los valores, consideramos relevante enfatizar el aspecto de los valores éticos para definir un perfil docente. Aunado a lo anterior, es pertinente considerar las recomendaciones que aporta la Conferencia Internacional de Educación de la UNESCO (1998), citadas por Bar (1999) sobre el rol de los docentes en el cambiante mundo actual, las cuales señalan requerimientos de la sociedad para las próximas décadas.

\section{El perfil del docente y la docente de sétimo año}

Como se señaló anteriormente, el sétimo año es el nivel con mayor índice de deserción y de reprobación en nuestro país. Muchos apuntan que la razón que más incide en estos resultados es que se encuentra en un período de transición entre la primaria y la secundaria. Castro et al (2007) señalan que los y las estudiantes le dan gran relevancia al(a) docente de secundaria. Los y las docentes afirman que la forma de explicar (su metodología) y las relaciones interpersonales (de acompañamiento y de respeto) que establecen con sus estudiantes, determinan que a un(a) docente se le considere eficiente o no, ya que de estos dos factores depende su permanencia en la educación secundaria.

Cabe preguntarse:

1. ¿Cuáles son las condiciones sociales, políticas y culturales en las que trabajan, actualmente, los y las docentes de secundaria? 
2. ¿Se debe especializar a los y las docentes de secundaria en determinados niveles educativos (octavo, undécimo, por ejemplo)?

3. ¿Está todo el profesorado consciente de la realidad educativa del sétimo año?

4. ¿Están los y las docentes de secundaria preparados para mediar el proceso educativo en sétimo año?

5. ¿Requiere el sétimo año de un o una docente con características particulares?

6. ¿Qué características, saberes, habilidades y destrezas debe tener el profesorado de sétimo año para incidir en la permanencia de los y las estudiantes de este nivel educativo?

Sin duda, para responder a cada una de estas interrogantes se necesita de una profunda reflexión, que no puede partir únicamente de supuestos teóricos, sino que, requiere de la investigación en los diferentes contextos educativos. Los profesores y las profesoras en ejercicio son quienes, a partir de la lectura de su realidad educativa, desde la gestión y la vida interna de las aulas de secundaria, pueden caracterizar cada uno de los procesos que se dan en la educación secundaria y, particularmente, en el sétimo año. Contestar a estas preguntas implica construir el perfil de los y las docentes de este nivel.

Esta necesaria construcción del perfil, demanda la participación del estudiantado, de los y las docentes, de los directores y las directoras de centros educativos de secundaria, de funcionarios y funcionarias del Ministerio de Educación Pública, y del personal académico de las instituciones formadoras de docentes, quienes, en consenso, analizarían todo el bagaje de saberes conceptuales, procedimentales y actitudinales que requieren los y las docentes de sétimo año en su práctica pedagógica actual y futura.

Esta construcción implica, también, cuestionarse sobre la calidad de nuestro sistema educativo. Se afirma que la calidad de un sistema educativo está directamente relacionada con la calidad de sus docentes. La calidad implica que nos cuestionemos sobre qué es lo que se está haciendo, por qué se hace así, qué implicaciones tiene lo que se hace, qué virtudes y debilidades se tienen y cómo se puede mejorar. Se debe trazar un objetivo claro, una visión de 
futuro y a lo que se quiere llegar, para lo que se necesita señalar una ruta con metas bien definidas y senderos claros.

Si se desea universalizar la educación secundaria, como lo afirman especialistas del Estado de la Educación Costarricense (2005), se debe identificar estrategias para que el o la estudiante de sétimo permanezca en el colegio.

El perfil del educador de este nivel educativo permitirá poner en marcha un conjunto de mecanismos que den seguimiento al proceso de aprendizaje de los estudiantes.

El Ministerio de Educación Pública podrá definir, claramente, qué tipo de educador y de educadora requiere contratar. Indicará al profesorado qué es lo que requiere y por qué. Asimismo, los directores de instituciones educativas de secundaria evaluarán el desempeño de estos docentes, de acuerdo con criterios definidos de antemano y conocidos por la comunidad educativa.

Las universidades incluirían en los procesos de formación inicial de docentes, el conjunto de saberes que les serán exigidos a los educadores y las educadoras en su práctica profesional $y$, se enfatizaría en aquellos elementos que todo profesor y profesora de sétimo requiere para mediar los procesos de aprendizaje de sus estudiantes. El perfil docente ofrecerá una guía acerca de lo que se debe perseguir no sólo en lo académico, sino, también, en lo personal y en lo humano. Esto, necesariamente, lo llevará a innovar y a implementar estrategias docentes.

Definitivamente, quienes más se beneficiarían de la construcción del perfil docente, serían los y las estudiantes de sétimo año, puesto que se les ayudaría, de forma más adecuada, en la transición de primaria a secundaria, se les acompañaría en su aprendizaje, permanecerían en el sistema, aprobarían, se promoverían a los siguientes niveles y podrían optar por un futuro mejor al que les habría esperado, si sólo tuvieran la primaria completa.

\section{Consideraciones finales}

Existen diferentes razones que justifican la necesidad de construir el perfil profesional docente de sétimo año. 
Primero, el sétimo año como el eslabón inicial en la educación secundaria de nuestro país, define, en la mayoría de las ocasiones, si un estudiante continúa o no en el sistema educativo, con las implicaciones sociales que esto tiene.

Segundo, la conformación de un perfil del y de la docente de sétimo año tendría una incidencia directa sobre los procesos de enseñanza y de aprendizaje, porque habría una mayor claridad sobre lo que ese y esa docentes requieren, no sólo en lo teórico-práctico, sino también en lo didáctico-metodológico.

Tercero, ese perfil puede aportar las características, las cualidades y los principios idóneos para un docente que labore con este tipo de población; lo que le permitirán un mayor acercamiento a sus estudiantes; de modo que entienda lo que pueden aprender los jóvenes en este año escolar, según su madurez física y emocional, la forma en que aprenden y, con ello, el beneficio directo de la permanencia en el sistema y su promoción dentro de éste.

\section{Referencias}

Bar, G. (1999). Perfil y competencias del docente en el contexto institucional Educativo. Versión electrónica. Trabajo presentado en el I Seminario taller sobre Perfil del docente y estrategias de formación, realizado en Lima, Perú. Recuperado el 12 de junio de 2007, de http://www.oei.es/de/gb.htm

Castro, M., Díaz, M., Fonseca, H., Moraga, L., Umaña, W. y Ruíz, S. (2007). Factores de éxito en la transición de 60 a 7o año. Heredia, Costa Rica: Universidad Nacional.

Costa Rica. Ministerio de Educación Pública. (2001). Estructuras curriculares III Ciclos y educación diversificada (todas las modalidades). San José: Autor.

Díaz Barriga, F. y Hernández, G. (2002). Estrategias docentes para un aprendizaje significativo. Una interpretación constructivista. (2ª ed.). México: Editorial Mc-Graw Hill.

Papalia, D., Wendkos, S. y Duskin, R. (2005). Desarrollo humano. (9a ed.). México: Editorial McGraw-Hill. 
Proyecto Estado de la Nación en Desarrollo Humano Sostenible. (2005). Undécimo informe. Estado de la Educación Costarricense Estado de la Nación en Desarrollo Sostenible. San José, Costa Rica: Proyecto Estado de la Nación.

Proyecto Estado de la Nación en Desarrollo Humano Sostenible. (2006). Duodécimo informe. Estado de la Nación en Desarrollo Sostenible. San José, Costa Rica: Proyecto Estado de la Nación.

Ramos, M. (1996). Perfil del docente hoy y su rol de facilitador humanista. Facultad de Ciencias de la Educación, (13), 6, 341-358.

Recio, A. (1993). El perfil del educador para el Siglo XXI. Tablero, 46, 22-27.

Ruíz, A. (2006). Universalización de la educación secundaria y reforma educativa. San José: Editorial de la Universidad de Costa Rica-CONARE.

Shön, D. (1998). El profesional reflexivo. Barcelona: Paidós.

Valenzuela, A. (1996). Perfil profesional del educador para la enseñanza media. Perspectiva educacional, 26, 117-126. 
